Clio. Femmes, Genre, Histoire

2 | 1995

Femmes et Religions

\title{
La Vierge mise à nu par ses chambrières
}

\section{Marlène Albert-Llorca}

\section{Q OpenEdition}

Journals

Édition électronique

URL : https://journals.openedition.org/clio/494

DOI : $10.4000 /$ clio.494

ISSN : 1777-5299

Éditeur

Belin

Édition imprimée

Date de publication : 1 novembre 1995

ISBN : 2-85816-283-2

ISSN : 1252-7017

\section{Référence électronique}

Marlène Albert-Llorca, "La Vierge mise à nu par ses chambrières ", Clio. Histoire, femmes et sociétés

[En ligne], 2 | 1995, mis en ligne le 01 janvier 2005, consulté le 29 avril 2022. URL : http://

journals.openedition.org/clio/494 ; DOI : https://doi.org/10.4000/clio.494

Ce document a été généré automatiquement le 29 avril 2022.

Tous droits réservés 


\title{
La Vierge mise à nu par ses chambrières
}

\author{
Marlène Albert-Llorca
}

1 Dans la procession célébrée en l'honneur de la Vierge dels Desemparats, patronne de Muro del Alcoy, neuf femmes vêtues d'une robe noire et coiffées d'une somptueuse mantille de dentelle de la même couleur défilent dans le cortège juste avant la statue. Elles doivent cette place privilégiée à leur fonction: la confrérie de la Vierge les a élevées pour un an au titre de camarera, qui les engage à entretenir la chapelle de la sainte patronne pendant l'année en cours et leur donne, en échange, le droit de l'habiller ou, plus exactement, de la changer pour sa fête. Comme toutes les statues du Pays valencien qui sont l'objet d'une vénération particulièrement intense, la Vierge de Muro del Alcoy possède en effet plusieurs jeux de vêtements qu'elle porte à tour de rôle pendant l'année. Quelques jours avant sa fête, ses caméristes se sont enfermées dans sa chapelle pendant plusieurs heures pour lui ôter son «habit de tous les jours » et lui mettre ses plus beaux bijoux et sa tenue de gala - une tunique et un manteau de couleur blanche, ornés de ramages brodés au fil d'or, qu'une dizaine d'amples jupons, soigneusement amidonnés, permettent de tenir en forme.

2 La coutume de vêtir les statues de la Vierge est fort ancienne. Dès le XIII ${ }^{e}$ siècle, en Espagne, certaines d'entre elles possédaient des couronnes et des capes, appelées mantos ou mantells. A partir du XVI siècle, leur garde-robe s'enrichit sensiblement. Un inventaire des biens de la chapelle de la Vierge del Remey (du Remède), vénérée près de la ville de Vich (Catalogne) mentionne en 1586 plusieurs bijoux (une couronne, des chaînes et des médailles, des boucles d'oreille, etc.), vingt-deux mantells, trois devants (litt. vêtement de devant) et un cosset (corsage ou corset) ${ }^{1}$. Les documents conservés en Italie suggèrent également que les statues étaient entièrement habillées dès cette époque et cela est confirmé, pour le siècle suivant, par les textes des synodes et des conciles qui condamnent l'existence d'effigies destinées à être vêtues : " que les dites images, précise par exemple une constitution synodale publiée à Vich (Catalogne) en 1628 , soient faites désormais de bulto ${ }^{2}$, comme on dit vulgairement, et qu'en aucune 
manière elles ne soient sculptées tout à fait nues, notamment dans leurs parties honteuses $»^{3}$.

3 L'Église post-tridentine s'est également préoccupée de légiférer sur le style des vêtements portés par les statues - plusieurs textes précisent qu'il est interdit de les accoutrer de façon profane - mais elle n'a pas fixé, en revanche, de règles uniformes régissant leur habillage, ni l'identité de ceux qui l'accomplissent, et cela tient au statut des images cultuelles dans le catholicisme. L'Église ne leur reconnaît pas la sacralité sui generis qui est attachée aux reliques des saints; elles ne sont pas non plus, comme le pain eucharistique, soumises à un rituel de consécration. Aussi les règles qui régissent leur production et leur manipulation sont-elles moins strictement définies qu'elles ne le sont dans d'autres religions ${ }^{4}$. L'art religieux occidental n'est pas, on l'a souvent souligné, un art sacré. La confection des images saintes n'est pas réservée à un corps d'artisans spécialisés et les artistes jouissent d'une certaine liberté dans l'exécution des commandes qui leur sont faites, au moins sur le plan stylistique. L'entretien des images cultuelles, d'autre part, a presque toujours été confié à des laïcs, sacristains ou membres d'une confrérie locale. Bien des tableaux ou des statues, au demeurant, appartiennent à une de ces pieuses associations, y compris lorsqu'elles sont conservées dans l'église paroissiale. C'est le cas, en Espagne, des Christs et des Vierges qui sortent en procession pendant la Semaine Sainte : il est à peine utile de préciser qu'elles sont habillées, le cas échéant, par les membres des confréries placées sous leur patronage.

4 L'image de la Vierge-patronne d'une localité a, en fait sinon en droit, un statut un peu différent et cela explique que le clergé se soit parfois réservé le droit de l'habiller ou, du moins, de participer à l'opération. C'était le cas au Moyen-Age à Tournai où les prêtres changeaient le manteau de la Vierge une fois par $a^{5}$; il en est toujours ainsi à Mende où la Vierge Noire dédicataire de la cathédrale est théoriquement habillée par le prêtre, assisté par la sacristine ${ }^{6}$. Dans le Pays valencien, en revanche, la statue de la Viergepatronne est toujours habillée par des femmes, aujourd'hui du moins. Cela ne signifie pas que les habitants de cette région considèrent cet acte comme une manipulation technique sans importance. La valeur qui lui est accordée se mesure au contraire aux règles coutumières qui le régissent : personne, hormis ses habilleuses, n'a le droit de voir les vêtements de la Vierge en dehors des périodes où elle les porte et son habillage se déroule toujours à huis clos - le prêtre lui-même ne peut y assister. Ces interdits manifestent parfaitement ce qu'est la statue pour les fidèles: un objet sacré, qui ne peut être vu et touché par n'importe qui. Cette conception, cependant, rend énigmatique l'attribution à des femmes de la fonction de camériste : elles sont écartées de la prêtrise et, plus généralement, de toute tâche obligeant à manipuler le sacré. Comment comprendre donc que la coutume leur accorde le droit de changer la Vierge et donc de voir et de toucher son "corps » même? Comment les titulaires de cette charge l'assument-elle et quelle signification lui accordent-elles?

6 Les habitants du village d'Agres mettent en scène chaque année, dans la nuit du 31 août, ce qu'ils appellent «l'apparition» de leur Vierge. Entendons l'apparition de la statue qu'un berger, Gaspard Thomas, découvrit entre les branches d'un des micocouliers qui poussent devant son ermitage: elle lui ordonna d'aller dire aux habitants du village qu'elle voulait être leur reine et lui rendit son bras (Gaspard était manchot de naissance) pour manifester son pouvoir. Une des versions orales de la légende ajoute que les autorités du diocèse décidèrent alors de faire porter la statue à 
Alicante, capitale de la Province : elle revint d'elle-même sur le lieu de son épiphanie. La Vierge d'Agres était déjà devenue célèbre pour ses miracles quand une épidémie de peste se déclara dans la région. Les habitants d'Ontenyente, une ville voisine atteinte elle aussi par le fléau, demandèrent à ce qu'elle soit conduite en procession jusqu'à eux. Arrivée à la limite de la commune d'Agres, la statue s'alourdit au point qu'il fallut renoncer au projet. Dans toutes les villes du Pays valencien qui se sont vouées à la Vierge, il existe des légendes analogues. Elles expriment parfaitement ce qu'est la statue de la sainte patronne : non pas une «image » mais une personne, capable de se déplacer, de parler, etc. ; pas même une incarnation de « la » Vierge Marie, mais « une » Vierge $^{7}$ qui a choisi de se donner à une ville et pas à l'ensemble du peuple chrétien ni, a fortiori, à l'Église.

7 La Vierge-patronne appartient à la cité et cela explique, en partie tout au moins, que le droit de l'habiller ne revienne pas au curé. Il est même assez rare, aujourd'hui du moins, qu'il soit chargé de nommer les caméristes de la Vierge. Cette fonction, dans certaines localités, est attachée à quelques familles où elle se transmet de mère en fille (ou, à défaut, de la tante à l'aînée de ses nièces) et ses titulaires, dans ce cas, s'occupent aussi de garder et d'entretenir les mantos et les tuniques de la statue. Elles doivent certainement ce double privilège à leur position sociale. L'article qui définit la charge de camarera dans le règlement de la confrérie andalouse de la Vierge de Setefilla, édité en 1926, précise en effet que les chambrières doivent appartenir à des familles disposant de l'espace et des moyens nécessaires à la conservation et à l'entretien des vêtements de la Vierge ${ }^{8}$. Il ne stipule pas, en revanche, que la fonction soit héréditaire mais on comprend qu'elle ait pu le devenir, notamment dans les petites bourgades où peu de familles pouvaient satisfaire aux exigences mentionnées. Ce mode d'accession au titre de camarera, cependant, est devenu relativement rare dans le Pays valencien et, là où il existe encore, il suscite de vives protestations : « la Vierge appartient à tous et il n'y aucune raison que ce soient toujours les mêmes qui l'habillent ", m'ont dit en substance plusieurs femmes. Aussi la plupart des villes ont-elles adopté (parfois à une date récente) la règle en vigueur à Muro del Alcoy : les camareras y sont élues pour un an par la confrérie de la Vierge. La quasi totalité des familles, précisons-le, sont représentées à l'intérieur de cette association par au moins un de leurs membres : à travers la confrérie, c'est donc la cité tout entière qui désigne les camareras.

8 Accéder à ce titre est un honneur fort onéreux : il n'est pas rare que la titulaire dépense plus de 10000 francs pour satisfaire à ses obligations (acheter le costume emblématique de la fonction, faire un don à la chapelle de la Vierge, offrir un repas aux autorités civiles et religieuses pendant la fête, etc.). Mais la perspective de ces charges financières ne décourage pas les candidates : à Biar, une petite ville de 3500 habitants où la confrérie élit chaque année quatre caméristes, il a fallu ouvrir une liste d'attente où certaines candidates se sont inscrites il $\mathrm{y}$ a plus de dix ans. Interrogées sur leurs motivations, certaines répondent qu'elles en ont fait le vœu. Mais la plupart demandent à être caméristes " parce que toutes celles qui l'ont été disent que c'est très émouvant ». Et toutes les femmes qui ont vécu cette expérience - une seule fois ou pendant plusieurs années - l'ont, en effet, évoquée au cours de nos entretiens avec une voix cassée par l'émotion et, parfois, des yeux embués par les larmes.

Dans la chambre de la Vierge

Les connotations du terme camarera permettent de cerner quelques composantes de ce sentiment, souvent déclaré « inexplicable » par les caméristes que j'ai interrogées. Dans 
l'Espagne d'Ancien Régime, on appelait camareras les dames d'honneur d'une princesse ; la reine avait en outre une camarera mayor, sa première femme de chambre. Et c'est en effet comme une reine, ou plus exactement comme la reine de la cité, que l'on traite rituellement la Vierge-patronne. À Valence, la Vierge dels Desemparats a non seulement sa camarera mayor mais aussi sa corte de honor, composée de mille femmes qui, tout au long de l'année, se relaient deux à deux pendant la journée pour prier devant elle. Partout, la statue porte une couronne somptueuse qui réunit l'or et les bijoux offerts par les habitants de la ville. Généralement conservée dans une chapelle située hors de l'agglomération, elle y descend pour sa fête : les autorités religieuses, civiles et militaires viennent l'accueillir aux portes de la cité dont les balcons ont été drapés de tissus pour l'occasion, comme on le faisait autrefois lors des Entrées royales.

11 On conçoit que les habilleuses de la Vierge soient particulièrement émues d'avoir apprêté Celle à qui s'adressent ces honneurs. Cette émotion comprend une part d'inquiétude - «il faut faire attention aux plus petits détails. Regarder si ça tombe bien, si tout est comme il faut » - et une immense fierté, que les caméristes n'ont pas toujours consenti à avouer aussi ingénument que l'habilleuse actuelle de la Vierge de SaintLaurent de Cerdans, dans les Pyrénées catalanes:

Ça m'a plu de le faire, oui... Parce que, des fois, on lui dit: - Quand même, tu pourrais nous dire merci, tu pourrais nous dire quelque chose, on lui dit. Quand elle nous regarde, comme ça : - Quand même, tu pourrais nous dire quelque chose. On a l'impression qu'elle nous regarde, et alors on lui dit: - Quand même, tu pourrais nous dire merci.

12 Habituellement, ce sont les hommes qui dépendent du bon vouloir des divinités. L'habillage, dans une certaine mesure, renverse ce rapport. La Vierge n'est elle-même qu'une fois habillée et elle est, en ce sens, ce qu'en font ses caméristes. Aussi se montrent-elles très jalouses de leur pouvoir :

Avant, il y avait la camarera mayor. C'était la plus ancienne : avant la guerre, elle était déjà camarera (...). Mais, même quand elle est devenue trop vieille pour pouvoir habiller la Vierge, elle a voulu continuer à venir avec nous. Elle s'asseyait et "Ça, ce n'est pas comme ça" ou "Ça, il faut le faire comme ça".

13 Tout aussi significatif, le conflit qui a éclaté à Hondón de las Nieves lorsque la Présidente de la confrérie de la Vierge a proposé d'adopter le principe de rotation des camareras en vigueur dans d'autres localités : deux des quatre caméristes en exercice ont immédiatement quitté la confrérie.

Mais l'émotion éprouvée par les caméristes vient aussi, et surtout, de ce que leur fonction de camarera (le terme dérive de cámara, la chambre) les amène à pénétrer dans l'intimité de la Vierge :

Moi, je l'avais toujours vue dans les hauteurs. Et alors, de la voir comme ça... La première impression que j'ai éprouvée, c'est un immense respect et aussi la peur de la toucher, au cas, je ne sais pas moi, au cas où ça lui ferait mal. C'est comme si on risquait de lui faire mal.

15 Toujours située au-dessus du maître-autel, la statue de la Vierge est visible toute l'année mais elle ne peut être approchée qu'en de rares occasions, lorsqu'on la descend de ses «hauteurs » le jour de la fête patronale ou lorsque les fidèles sont autorisés à monter dans son camarín, sa "petite chambre ». La «niche » où elle se dresse est en effet une chapelle construite au-dessus du maître-autel et ouverte sur l'église de façon à laisser voir la statue ${ }^{9}$. On y accède par deux escaliers latéraux, l'un destiné à la montée et l'autre à la descente. Ce dispositif permet de rendre l'image accessible aux 
fidèles, tout en limitant l'affluence dans le camarín (l'escalier qui y mène est des plus étroits). Le prêtre peut ainsi contrôler leurs gestes : les fidèles ont seulement le droit de toucher le bas du manto ou de la robe de la Vierge.

Les caméristes, en revanche, peuvent voir et toucher son "corps » ou ce qui en tient lieu. Ces "statues ", en effet, sont parfois formées d'un bâti de bois, de forme conique, où seuls le buste, les bras (généralement articulés) et la tête sont façonnés. Il peut s'agir aussi de mannequins tout à fait semblables à ceux qu'utilisent les magasins de confection. La rigueur du secret qui entoure l'habillage s'explique en partie par la crainte de révéler que la Vierge n'est, en réalité, qu'une de ces figures pitoyables que les antiquaires appellent si cruellement des «Vierges chauves ». "Je n'aimerais pas que quelqu'un soit là quand je change la Vierge, elle fait trop poupée », m'a confié la chambrière de la Vierge des Sept Douleurs, à Perpignan. Aussi accorde-t-on rarement, aux camareras élues pour un an, le droit de voir la Vierge entièrement dénudée. À Muro del Alcoy, par exemple, elles n'ont plus que les bijoux à mettre quand elles arrivent: "Ce sont toujours les mêmes qui l'habillent. La Vierge n'est pas une imagen de bulto, et alors elles ont peur que nous nous moquions d'elle ». À Biar, on concède seulement aux caméristes, ici appelées mayordomas ${ }^{10}$, le droit de lui mettre son manteau et on prend soin, notamment, d'éviter qu'elles la voient sans sa perruque: «Ce serait trop impressionnant ", m'a dit le président de la confrérie. À Saint-Laurent de Cerdans, ce sont les caméristes elles-mêmes qui s'interdisent de la voir sans cheveux : «C'est une femme qui lui a donné sa chevelure. Nous ne l'avons jamais enlevée, jamais. Nous la coiffions, nous l'arrangions mais nous ne lui avons jamais enlevé ses cheveux, non ». La chevelure est un des signes les plus manifestes et les plus valorisés de la féminité et les femmes ressentent toujours la calvitie comme une expérience tragique. Aussi les Vierges habillées ont-elles une perruque de longs cheveux, soigneusement bouclés, qui sont souvent, comme à Saint-Laurent, de vrais cheveux. Les ôter à la Vierge, ce serait la dégrader ou, en d'autres termes, lui enlever un des éléments qui contribuent à transformer cet objet en sujet.

17 Les caméristes, pourtant, oublient visiblement, lorsqu'elles l'habillent (comme les petites filles qui jouent à la poupée ?), que la Vierge n'est qu'une chose: "C'est une statue mais, pour moi, ce n'est pas une statue ». La statue est la Vierge et c'est bien cette identité qui explique l'intensité de l'émotion de ses caméristes. Mais la Vierge, comme chacun le sait, est une femme: on ne pouvait donc laisser des hommes l'habiller et, surtout, la déshabiller. Les prêtres, il est vrai, se sont parfois réservé le droit de changer le manto posé sur ses épaules. Il n'est pas certain, cependant, qu'ils se soient jamais permis de la changer de pied en cap. C'est, du moins, ce que suggère ce témoignage concernant la ville de Palencia, au XVIII ${ }^{e}$ siècle :

Quand on habille Sa Majesté, on ne lui quitte jamais ses sous-vêtements. Elle porte toujours ceux qu'elle avait lorsqu'elle apparut; on change seulement ses vêtements de dessus. C'est toujours un prêtre qui l'habille et la pare de rubans et de bijoux. Néanmoins, quelques unes des femmes les plus importantes de la Ville assistent à cet acte pieux en signalant avec un roseau l'endroit où il doit mettre la ceinture ou les bijoux afin qu'ils ressortent mieux ${ }^{11}$.

18 Les femmes (et pas n'importe lesquelles), à Palencia, ne participaient à l'habillage qu'en tant que « conseillers techniques ». Seul le prêtre touchait la statue, ce qui permettait de conformer l'opération au privilège attaché à la fonction sacerdotale - manipuler le sacré. En même temps, la procédure adoptée permettait de satisfaire aux règles de la morale commune: une femme peut, sans se compromettre, voir une de ses compagnes 
dénudées mais pas un homme, même lorsqu'il est prêtre ${ }^{12}$. Et c'est en effet la pudeur qu'ont invoquée les femmes et, parfois, les prêtres, quand j'ai demandé pourquoi la Vierge était toujours habillée par des femmes. Bien rares, au demeurant, sont ceux - ou même celles - qui ont vu le «corps» de la Vierge : ses vêtements cachent souvent une statue tout à fait ordinaire; les mannequins destinés à être habillés, quant à eux, portent presque toujours une chemise peinte qui descend jusqu'aux genoux, voire jusqu'aux chevilles.

19 La pudeur, cependant, n'explique pas entièrement les modalités de l'habillage, comme le suggère ce témoignage, recueilli l'an passé à Saint-Laurent de Cerdans auprès d'une des caméristes qui ont servi jusque dans les années 1980 la Vierge de la Sort, dédicataire d'une chapelle située à l'extérieur de l'agglomération :

- Ah! nous prenions des précautions! Nous nous arrangions pour ne pas voir comment la Vierge était faite : à mesure que nous faisions descendre la chemise, nous enfilions l'autre. La Vierge était placée sur une espèce de tréteau, mais nous ne l'avions jamais vue. Nous l'avons découvert quand monsieur le curé a fait faire la restauration du socle.

Q. - Et donc, vous ne saviez pas que la Vierge était vide?

- Si, parce que nous le sentions en la touchant. Mais nous faisions tout pour ne pas voir.

Les caméristes de la Vierge de La Sort savaient que l'effigie n'avait pas de corps et elles pouvaient même s'imaginer assez précisément comment elle était faite. En effet, elles changeaient aussi, avant la Semaine Sainte, la Vierge des Sept Douleurs : « Là, nous lui enlevions tout, et nous voyions un buste et, puis, le reste, c'était un truc en bois ». L'effigie de la Vierge des Sept Douleurs est posée sur un autel d'une chapelle latérale du sanctuaire ; Notre-Dame de la Sort a son camarín au-dessus du maître-autel et elle est manifestement considérée comme « la » Vierge de Saint-Laurent, même si elle n'en est pas la sainte patronne. Les deux images n'ont pas le même degré de sacralité et la différence de comportement des caméristes à leur égard contribue à le montrer. Elle montre aussi que leurs "précautions » n'étaient pas motivées par la pudeur et cela est apparu, au cours du même entretien, lorsque mon interlocutrice m'a raconté comment la tradition explique le nom de la Vierge de Coustouges, un village situé à cinq kilomètres de Saint-Laurent :

On dit qu'elle est assise sur une aubépine. On le dit. Personne ne le sait, non plus, parce que personne ne l'a jamais regardée. Mais on le dit et c'est pourquoi on l'appelle la Mare de Déu del Espinas [Notre-Dame de l'aubépine]. Et on dit que celui qui regarderait si elle est vraiment assise sur une aubépine deviendrait aveugle. On dit : - Ça, ne le faites jamais.

On comprend que les caméristes de Notre-Dame de la Sort se soient interdit de dénuder leur Vierge : elles ne pouvaient sans doute s'empêcher de craindre le châtiment promis aux femmes trop curieuses dans le village voisin! Cette peur d'être sacrilège fait certainement partie intégrante de l'émotion de toutes les caméristes, même si elle n'est pas justifiée (au moins explicitement) par la crainte d'une punition: «On est trois à l'habiller. Mais, si j'étais toute seule, je n'irais pas. J'aurais peur ", a avoué l'une d'elles après l'entretien enregistré ; « La première fois, eh bien, cela m'a causé une impression forte, parce que je ne croyais jamais, tu sais, je ne croyais jamais... Il y en a qui disaient : "C'est un squelette » ou "C'est un... ». Chacun disait quelque chose de différent. Et tu la vois et tu sais ce que c'est, tu l'as vu. C'est un buste, et puis après... comme une jupe ». 

1383 qui donne aux Pinzocchere della Calle del Pestrin le droit d'habiller et de servir la Vierge. Les pinzocchere étaient des femmes - généralement veuves - qui se chargaient, entre autres œuvres pieuses, d'accompagner les cortèges funéraires; tout en vivant dans le siècle, elles suivaient la règle des frères mendiants, dont elles portaient l'habit ${ }^{13}$. Cette pieuse association n'existe plus aujourd'hui mais la coutume de San Stefano a gardé une trace de son existence : dans les années 1930, seules les veuves les plus âgées de la paroisse avaient le droit de changer la statue de la Vierge des Sept Douleurs. San Stefano n'est pas un cas absolument unique : à Augusta, en Sicile, la Vierge des Sept Douleurs était également habillée par des femmes d'un certain âge; à Altamura, dans les Pouilles, par des femmes vêtues de noir ${ }^{14}$. On se souvient que les camareras du Pays valencien portent elles aussi un costume noir : cet usage est peut-être une manière de perpétuer symboliquement une règle qui a existé autrefois, ici comme en Italie. À Elche, en effet, au XIXe siècle : «seules les camareras les plus âgées et issues des meilleures familles peuvent changer les sous-vêtements de la Vierge ; pendant qu'elles officient, les jeunes filles se retirent dans un coin de la pièce $»^{15}$. On pourrait être tenté, ici encore, d'invoquer la pudeur : on accorde plus aisément à une femme âgée qu'à une jeune fille le droit de voir une autre femme dévêtue. Mais est-ce leur âge qui qualifiait les candidates à la charge de caméristes ou plutôt le fait qu'une femme âgée est souvent une veuve? Dans les premières décennies de ce siècle, en Italie, les habilleuses de la Vierge devaient être, en effet, soit des femmes âgées ou des veuves, soit des religieuses ou des jeunes filles ${ }^{16}$, c'est-à-dire des femmes écartées, par leur âge ou leur condition, de la sexualité.

Dans le Pays valencien, aujourd'hui, cette exigence semble avoir presque partout disparu : les camareras sont généralement des femmes mariées. Les caméristes que j'ai interrogées ont, par ailleurs, toujours affirmé qu'elles ne se soumettaient à aucune prescription rituelle avant d'habiller la Vierge: «Nous récitons une prière avant de commencer, c'est tout ». À Hondón, cependant, l'une d'elles a ajouté : «Nous mettons un tablier blanc. C'est un tablier que nous gardons, moi et les autres aussi, uniquement pour habiller la Vierge. Nous ne l'utilisons pour rien d'autre».

Cet acte pourrait sembler insignifiant si l'on ne savait que le statut des femmes s'inscrivait autrefois sur leur costume et, notamment, sur leur tablier : tissus, motifs décoratifs et couleurs variaient selon la région et la condition sociale, mais aussi selon l'âge de la vie ${ }^{17}$. Il est à peine nécessaire, par ailleurs, de rappeler les connotations de la couleur blanche dans notre culture : emblème de la pureté, elle est, bien entendu, une des couleurs de la Vierge Marie, notamment dans les représentations qui évoquent la doctrine de la Conception Immaculée. Or, la patronne d'Hondón n'est pas une Vierge- 
mère ${ }^{18}$ et elle s'appelle, de plus, Mare de Déu de les Neus - Vierge des Neiges. Cette identité a certainement contribué à imposer à ses caméristes l'idée qu'elles devaient être pures - réellement ou symboliquement - pour pouvoir toucher la statue. La même exigence, cependant, sous-tend, comme on l'a vu, les règles de désignation des caméristes italiennes : les veuves, les religieuses et les jeunes filles ont en commun de ne pas être souillées par les rapports sexuels.

Mais, mettre un tablier blanc, ce n'est peut-être pas seulement se «laver» de la souillure causée par les rapports sexuels. Ce peut être aussi se redonner symboliquement une virginité perdue ou encore dénier la présence du sang menstruel, un sang qui rend les femmes à la fois vulnérables et susceptibles de souiller ce qu'elles touchent ou voient ${ }^{19}$ : le tablier des jeunes filles, en effet, était souvent orné de broderies florales de couleurs vives ${ }^{20}$, euphémisme de ces autres "fleurs» dont l'apparition marque la fin de l'enfance. L'exigence de pureté peut donc être interprétée de façon plus ou moins stricte: de là, me semble-t-il, l'existence des différences présidant en Italie au choix des caméristes. On peut les élire parmi les femmes âgées, c'est-à-dire (on peut du moins le supposer) parmi les femmes ménopausées; parmi les jeunes filles, si l'on identifie pureté et virginité; parmi les veuves, si l'on prend seulement en compte la question des rapports sexuels. Les religieuses, quant à elles, peuvent appartenir à l'une de ces trois catégories. Du moins dans les faits. Symboliquement, ces «épouses " du Christ se considèrent (et se comportent) comme des jeunes filles ${ }^{21}$ que la vie monastique s'efforce de purifier entièrement - ce qui se traduit parfois par une aménhorrée bien réelle : en ce sens, elles sont certainement les meilleures candidates à la fonction de camériste. Mais les images mariales, répétons-le, n'appartiennent pas à l'Église et les communautés locales ou les confréries ont donc assez rarement confié à des religieuses le soin de les habiller. De fait, en Italie du moins, cette fonction a été préférentiellement accordée à des jeunes filles. Comment comprendre ce choix?

La Vierge Marie est la mère du Christ et c'est ce titre qui, aux yeux de l'Église catholique, justifie son culte. Mais cette mère est une vierge et ce sont habituellement les jeunes filles qui sont vierges. La virginité de Marie invitait donc à la considérer comme une éternelle jeune fille et cet aspect de son identité n'a rien de secondaire, comme le montrent certaines formes du culte marial - officielles ou paraliturgiques. La consécration à la Vierge du mois de mai, mois consacré depuis le Moyen-Age au courtisement rituel, est un des indices les plus clairs de son importance. L'Église, certes, ne l'a institué qu'au début du XVIII siècle, dans l'intention, semble-t-il, de lutter contre les « abus » des coutumes de mai. Mais elle n'aurait pu songer (ni réussir) à le faire si la Vierge n'avait été depuis longtemps déjà associée à cette période. On sait, notamment, que certaines confréries de la Vierge du Rosaire, au XVI e siècle, avaient choisi le mois de mai pour fêter leur sainte patronne : c'était le cas, notamment, dans certaines parties de la Catalogne ${ }^{22}$. Cette coutume s'est perpétuée juqu'à nos jours, alors que la fête liturgique du Rosaire a été fixée, en 1572, au premier dimanche d'octobre ${ }^{23}$.

Le mois de mai est en effet le mois des fleurs et le rosaire est une couronne de roses "spirituelles". La métaphore s'ancre dans des usages très communs à l'époque médiévale. Les jours de fête, les hommes et les femmes aimaient à se coiffer de « chapels » ou « chapelets » de fleurs; ces fragiles parures pouvaient aussi être données en dot à des jeunes filles (les futures " rosières ») ou en redevance à un Seigneur; on les offrait, enfin, comme gages d'amour. Or, la rose a été, dès l'époque médiévale, 
considérée comme la reine des fleurs : on ne peut donc s'étonner qu'on ait songé à couronner les statues de la reine des cieux de « chapels » de $\operatorname{roses}^{24}$, puis à lui offrir des rosaires. Ce lien métonymique se double d'une relation métaphorique: figurer la Vierge dans un rosier, comme on le fait parfois dans l'iconographie du Rosaire ${ }^{25}$, c'est sans nul doute suggérer qu'elle est sa plus belle fleur. Cette identification est explicite dans les poèmes qui lui sont adressés. Pour célébrer la Vierge, les clercs ont repris la rhétorique de la lyrique courtoise, où la femme aimée est souvent comparée à une fleur : il en est ainsi, par exemple, dans le très célèbre Roman de la rose de Guillaume de Lorris, long poème allégorique où l'objet de la quête amoureuse est identifié à une rose, découverte dans un verger enclos de hauts murs et protégée par "chardon aigu et poignant (...) Espines tranchanz et agües, Orties et ronces crochues $»^{26}$. La rose doit sans doute à son parfum et à sa beauté d'avoir été choisie pour évoquer la femme aimée, mais elle doit aussi son élection à ses épines : l'amour, pour les poètes courtois, n'a de prix que s'il est difficile (voire impossible) à conquérir. Aussi la rose est-elle devenue par la suite, comme l'a montré Yvonne Verdier, la fleur emblématique des jeunes filles, qui doivent savoir se barder d'épines jusqu'au mariage.

Si certaines confréries du Rosaire ont choisi de fêter leur sainte patronne pendant le mois consacré aux rites de courtisement des "jeunes filles en fleurs", c'est que la Vierge-rose peut être identifiée à l'une d'elles. La coutume de Jabaloyas, dans la province de Teruel, est tout à fait explicite à cet égard :

Dans la nuit du 30 avril, les garçons se réunissent dans une taverne aux alentours de dix heures pour y souper ensemble. Un peu avant minuit, ils se dirigent vers l'ermitage de la Vierge des Douleurs au son d'une jota. À minuit, ils mettent aux enchères le privilège de chanter en l'honneur de ladite Vierge (...). Les enchères terminées, le vainqueur chante un romance appelé los Mayos, qui décrit les perfections physiques d'une femme imaginaire; les autres reprennent en chœur après lui les différents couplets de la chanson ou seulement les deux derniers vers. Ce romance est chanté ensuite devant les maisons où vit une jeune fille courtisée par un des garçons du groupe. [Appelés mayos, comme les chants rituels, les garçons doivent, les jours suivants, apporter un présent à leur bien-aimée]. Le mayo doit aussi décorer la demeure de sa maya de branches où il a suspendu des cerises, des mouchoirs et même des capes et elle doit en retour (ou, plus exactement, elle devait quand la coutume était encore vivante) lui offrir une chemise. Le mayo qui avait chanté pour la Vierge devait, quant à lui, orner son autel de branches de feuillages puisqu'il la considérait comme sa maya ${ }^{27}$.

Des coutumes similaires sont attestées dans la vallée d'Ager, au nord-ouest de la Catalogne, où les garçons allaient chanter la première aubade de mai à la Vierge du Rosaire; à Murcie, ils se disputaient l'honneur de décorer de feuillages la porte de son église et de chanter pour elle, comme ils le faisaient pour leur bien-aimée ${ }^{28}$; à Valence, le jour de la fête de la Vierge dels Desemparats, qui a lieu le deuxième dimanche de mai, les hommes lui lancent rituellement des piropos (des compliments galants) et ses porteurs la font "danser" à la demande de la foule pendant son transfert de sa basilique à la cathédrale; dans d'autres villes de la région, on descend la Vierge de son ermitage au son d'une musique de valse. Les règles qui président au choix des porteurs de la Vierge de Biar, fêtée entre le 10 et le 13 mai, s'inscrivent dans la même logique. Pour accéder à cet honneur, il faut avoir fait son service militaire mais ne pas s'être fiancé, officiellement du moins: "Quand il est revenu du service, mon fils m'a dit: "Maman, je veux porter la Vierge parce que je veux lui dire adieu" (quiero despedirme de 
ella) ». On ne peut à la fois aimer une femme réelle et la jeune fille idéale - et située audelà de tout rapport charnel - que chantent les mayos.

La diffusion de la doctrine de l'Immaculée Conception ${ }^{29}$ et surtout de son iconographie a certainement contribué à mettre en valeur cet aspect de l'identité de la Vierge : Marie y est représentée sans enfant et, notamment après la Contre-Réforme, sous les traits d'une toute jeune fille. Or, c'est elle - et non plus la Vierge-Mère - qui se manifeste lors des grandes apparitions du XIXe siècle et du début du XXe : celle de la chapelle de la rue du Bac (origine de l'institution des Enfants de Marie), de La Salette, Lourdes, Fatima ou, plus récemment, San Damiano ou Medjugorge.

La plupart des Vierges-patronnes du Pays valencien, certes, sont des Vierges à l'Enfant. Mais personne ne parle jamais de celui-ci, comme s'il était un personnage tout à fait secondaire. Ses vêtements tendent même à le faire apparaître comme un double en réduction de sa mère : ils sont taillés dans le même tissu que ceux de la Vierge et ont exactement la même forme. Ce costume, il est vrai, n'est pas très féminin : l'Église de la Contre-Réforme a réussi à imposer presque partout en Espagne un type de vêtements dont la coupe et l'ornementation s'inspirent visiblement de celles des vêtements sacerdotaux ${ }^{30}$. Mais la féminité de la Vierge est cependant dénotée par ses dessous. Toutes les caméristes que j'ai rencontrées ont évoqué, avec un plaisir évident, l'abondance de ses jupons :

Sous sa robe, elle a quinze jupons! Ce sont des jupons très larges et bordés de dentelles, comme on en mettait autrefois. Ils sont plus ou moins longs et nous les mettons en étage, de façon à faire comme une pyramide. Pour que ça soit bien raide, nous les accrochons les uns sur les autres avec des épingles - il faut en mettre tout le tour pour que ça tienne bien - puis, nous mettons par-dessus le manto et la robe, que nous fixons aussi avec des épingles en faisant bien attention à ne pas faire de plis. C'est difficile parce que le tissu est très épais et, bien souvent, nous nous piquons avec les épingles.

La statue, extérieurement, a l'allure hiératique d'un prélat. Mais, dessous, elle est bien une femme et, plus précisément, une jeune vierge hérissée - comme il se doit d'épingles. Cet aspect de son identité est également manifesté, aux yeux de tous, cette fois, par sa longue chevelure. Seules les jeunes filles, en effet, sortaient autrefois avec les cheveux dénoués : après leur mariage, elles devaient les relever en chignon; depuis les années 1940, elles les coupent à ce moment-là. Or, la perruque de la Vierge est généralement formée de chevelures offertes à la suite de l'obtention d'une grâce ou "par dévotion » : n'est-ce pas, par conséquent, des cheveux de jeunes filles que porte la statue et celles qui les lui offraient n'entendaient-elles pas pérenniser ainsi leur état virginal, comme le font les épousées en lui laissant leur bouquet ou leur robe de mariée ${ }^{31}$ ?

Une jeune fille, en effet, n'est une jeune fille chrétienne que si elle est vierge, c'est-àdire semblable à la Vierge. On peut, me semble-t-il, mieux comprendre dans cette perspective le fait que l'on ait souvent réservé, en Italie, la fonction de camériste à des jeunes filles. Elles seules pouvaient la voir, comme l'ont vue, dans un autre contexte, Mélanie Calvat à La Salette ou Bernadette Soubirous à Lourdes. Sans doute pensait-on aussi qu'elles devaient la voir pour être de " vraies " jeunes filles.

Certains des textes synodaux ou conciliaires publiés dans la période de la ContreRéforme suggèrent que l'interdiction de fabriquer des effigies destinées à être habillées ne s'explique pas seulement par la volonté de «moraliser» les images saintes : l'Église aurait certainement souhaité abolir totalement la pratique de l'habillage ${ }^{32}$. Celle-ci 
institue en effet une différence entre les initiés qui peuvent la voir et la toucher, et les autres. Elle donne donc un pouvoir que les clercs ne pouvaient s'arroger (du moins pas totalement) parce qu'ils admettaient (et admettent encore) la double relation d'équivalence qui sous-tend le culte " populaire » des images : la statue est la Vierge et la Vierge est une femme. Ils devaient donc supprimer la coutume de l'habillage ou se résigner à laisser les femmes manipuler la Vierge à leur guise. On ne peut s'étonner qu'ils aient échoué. Les femmes ne pouvaient renoncer aisément à la possibilité de « faire » la Vierge et de se « faire » elles-mêmes à travers cette opération.

\section{BIBLIOGRAPHIE}

ALBERT-LLORCA, M.

1994. « La fabrique du sacré. Les Vierges “ miraculeuses ” du Pays valencien ». Genèses. Sciences sociales et histoire. $n^{\circ} 17$, sept. : 33-51.

1995. «Les fils de la Vierge. La broderie et la dentelle dans l'éducation des jeunes filles ». L'Homme, n 133, janv.-mars : 99-122.

AMADES, J.

1950. Costumari catalá. El curs del any. 5 vol. Barcelona, Ed. Salvat.

BOGATYREV, P.

1971. The Functions of Folk Costum in Moravian Slovakia. Paris, Mouton-La Hague.

CARO BAROJA, J.

1979. La estación de amor. Fiestas populares de mayo a San Juan. Madrid, Taurus.

CASSADOR, J.

1902. « Una questió iconológica. Les imatges vestides ». Revista de la Asociación Artístico-

Arqueológica Barcelonesa, Barcelona, vol. 3, 1901-1902.

DE LORRIS, G.

1967. Le roman de la rose, in Poètes et romanciers du Moyen-Age. Texte établi et annoté par A.

Pauphilet, Paris, « La Pléiade », NRF.

DUBY, G. et A.

1973. Les procès de Jeanne d'Arc. Paris, « coll. Archives », Eds Gallimard/Julliard.

DUVAL, A.

1991. Article « rosaire » in Dictionnaire de spiritualité ascétique et mystique. M. Viller éd., Paris, Beauchesne.

FAGNANI PAGAN, G. et BONARDO G.

1993. «Le Madonne "vestite" tra culto, storia e tradizione », in Madonne della Laguna. Simulacre da vestire dei secoli XIV-XIX. Sous la dir. de R. Pagnozzato, Roma, Instituto della Enciclopedia Italiana. 
FINE, A.

1984. «À propos du trousseau : une culture féminine? ", in Une histoire des femmes est-elle possible? Sous la dir. de M. Perrot, Marseille, Rivages : 156-188.

FUENTES Y PONTE, J.

1887 Memoria histórico-descriptiva del santuario de Nuestra Se-ora de la Asunción en la ciudad de Elche, Lérida, Imprenta mariana.

GORCE, M.

1931. Le rosaire et ses antécédents historiques. Paris, Ed. Picard.

KLAPISCH-ZUBER, C.

1990. La maison et le nom. Stratégies et rituels dans l'Italie de la Renaissance. Paris, Eds de l'EHESS.

NIERO, A.

1993. «Le Madonne « vestite » nella storia della pietà popolare », in Madonne della Laguna.

Simulacre da vestire dei secoli XIV-XIX. Sous la dir. de R. Pagnozzato, Roma, Instituto della

Enciclopedia Italiana.

PAGNOZZATO, R. (sous la dir. de)

1993. Madonne della Laguna. Simulacre da vestire dei secoli XIV-XIX. Roma, Instituto della

Enciclopedia Italiana.

TRENS, M.

1946. María. Iconografía de la Virgen en el arte espa-ol. Madrid.

TREXLER, R. C.

1991. « Habiller et déshabiller les images. Esquisse d'une analyse », in L'image et la production du sacré. Sous la dir. de F. Dunand, J.-M. Spieser, J. Wirth. Paris, Mérideins Klinsieck.

VERDIER, $\mathrm{Y}$.

1979. Façons de dire, façons de faire. La laveuse, la couturière, la cuisinière. Paris, Gallimard, «Bibliothèque des sciences humaines ».

\section{NOTES}

1. J. Cassador, $1902: 451$.

2. L'expression imagen de bulto (litt. image en volume) a d'abord désigné les statues par opposition aux peintures, puis les figures entièrement sculptées par opposition aux effigies destinées à être habillées : leur corps, en effet, se réduit souvent à un châssis de bois de forme conique.

3. Ce texte est cité par J. Cassador, op. cit. : 454. En Italie, il existait certainement bon nombre d'images « sculptées tout à fait nues » au XVIIe siècle puisqu'on voit apparaître à cette époque le terme biancheria (lingerie) dans les inventaires des biens des églises vénitiennes, seule région où une enquête systématique sur l'habillement des Vierges a été conduite, à la fois sur le plan historique et ethnographique (cf. R. Pagnozzato, 1993). 4. Ou, à l'intérieur même du monde chrétien, dans l'Église orthodoxe, où les images cultuelles sont considérées de plein droit comme des sacra.

5. Cité par R. Trexler, $1991: 198$. 
6. Je dois cette information à Annie Paradis, qui me l'a aimablement communiquée. Je remercie également Deborrah Puccio, qui m'a fait part du résultat de ses entretiens avec les caméristes de la Vierge de Gracia, patronne de Biar (Alicante). Mes propres enquêtes, conduites dans la région d'Alicante et dans les Pyrénées orientales, ont été financées par la Mission du Patrimoine Ethnologique.

7. Cf., pour une analyse plus développée du statut des images « miraculeuses » de la Vierge, M. Albert-Llorca, 1994.

8. Cité par R. Trexler, op. cit. : 229, note 95.

9. Le camarín, cependant, peut être fermé par un rideau, notamment lorsque les caméristes changent la statue. À Valence, il est fermé également lorsqu'on y célèbre un office " privé » (un mariage, par exemple) : la statue de la Vierge dels Desemparats, dans ce cas, est tournée vers l'intérieur du camarín. Ce dispositif, toujours dans cette ville, permet enfin de jouer « l'apparition » de la Vierge, le jour de sa fête (sur ce rituel, cf. l'article cité à la note précédente).

10. Les mayordomos sont, en principe, les dirigeants d'une confrérie. Les caméristes peuvent encore être appelées clavariesas, féminisation du terme clavario qui désignait le confrère chargé de garder les biens de l'association, notamment la statue de son saint patron.

11. Cité p. R. Trexler, op. cit. : 221.

12. Une femme peut également, sans déroger aux bonnes mœurs, vêtir un garçon, voire un homme, du moins si ce dernier est son fils. Cela explique, en partie, que les femmes aient pu s'octroyer le droit d'habiller, non seulement les Vierges mais aussi les images de l'enfant Jésus (cf., sur ce point, C. Klapisch-Zuber, 1990, ch. XIV) et les Christs de la Passion (si l'on admet qu'elles prenaient, dans ce cas, la place de Marie). L'habillage du Christ soulève cependant, comme l'a souligné R. Trexler (op. cit. : 205), la question de son ambiguïté sexuelle : à Perpignan, il porte une perruque faite avec des cheveux de femmes et, sous sa tunique, une chemise de femme.

13. D. Davanzo Poli, $1993: 124$.

14. A. Niero, $1993: 52 ; 54-55$.

15. J. Fuentes y Ponte, $1887: 129$.

16. A. Niero, op. cit. : 51-59.

17. Cf. P. Bogatyrev, 1971.

18. La statue a les mains jointes sur un bouquet de fleurs façonnées, m'a-t-on dit, avec de la mie de pain!

19. Y. Verdier, $1979: 150$.

20. P. Bogatyrev, op. cit. : 68-69.

21. Leurs travaux manuels - pâtisserie, broderie, fabrication d'images pieuses - étaient autrefois des occupations typiques des jeunes filles de bonne famille.

22. A. Duval, 1991 : col. 960. Il existe d'autres indices du privilège accordé au mois de mai dans le culte marial. Le hêtre où Jeanne d'Arc et ses compagnes allaient tresser des couronnes de fleurs pour la Vierge de Domrémy était l'arbre avec lequel on faisait le «mai » (G. Duby, 1973: 42). En 1449, la corporation des orfèvres de Paris avait coutume, le premier mai, d'offrir à Notre-Dame de Paris un arbre vert, le « mai verdoyant» (A. Certeux, RTP, IV : 262).

23. Cette décision fut prise après la bataille de Lépante : la victoire de la coalition chrétienne contre les Turcs fut attribuée à l'intercession de la Vierge du Rosaire. 24. Un couple de Tournai demande dans son testament, en 1406, que la statue de la Vierge soit couronnée d'un « cappiau de roses vermeilles » (M. Gorce, 1931: 88); en 
1458, les béguines de Lille s'engagent à fournir aux chanoines de Notre-Dame de la Treille « ung chappeau de roses ou de violettes ou d'autres verdures selon la saison, lequel chappeau sera offert et posé sur le chief de l'ymage de Nostre Dame en la chappelle que l'on dist a la Treille » (A. Duval, op. cit. : col. 942).

25. Il en est ainsi dans un tableau anonyme de la fin du XVIe siècle conservé au Musée de Picardie. Sur les rubans qui s'enroulent autour du corps de la Vierge, on lit: « Rose du ciel devant Dieu toute belle » et "Rose sans épines ». Sur une gravure illustrant un cantique catalan édité au XVIIe siècle, la Vierge est assise sur le tronc d'un rosier, dont les fleurs sont autant de médaillons historiés figurant les épisodes de l'Histoire Sainte offertes à la méditation des fidèles. Elle apparaît encore sur un rosier dans une peinture catalane du XVIe siècle qui représente la légende du chevalier de Cologne : poursuivi par ses ennemis, il se jeta aux pieds de la Vierge en récitant des Ave Maria, qui se changèrent en roses (M. Trens, 1946 : 308). Soulignons, par ailleurs, que la Vierge du Rosaire est appelée en Catalogne Mare de Déu del Roser (Vierge du Rosier).

26. G. de Lorris, $1967: 572$.

27. J. Caro Baroja, $1979: 69$.

28. J. Amades, 1950, III : 435 ; J. Caro Baroja, op. cit. : 104.

29. Proclamée comme dogme en 1854, elle figure dès 1439 parmi les « articles de foi » de l'Église catholique.

30. Comme le souligne, à juste titre, l'historien de l'art M. Trens, 1946 : 649.

31. La coutume de broder le trousseau en blanc peut être interprétée dans la même perspective (cf. A. Fine, 1984 ; M. Albert-Llorca,

32. C'est le cas de la constitution synodale de Vich dont j'ai cité un extrait au début de cette étude. Elle précise, après avoir notifié l'interdiction de fabriquer des images « tout à fait nues »: " afin qu'on n'ait pas besoin de vêtements pour leur ornement ». R. Trexler cite un texte de saint Jean d'Avila qui déclare également : « Il serait convenable qu'aucune image ne soit habillée » (op. cit. : 219, n. 3).

\section{RÉSUMÉS}

Les statues de la Vierge qui sont l'objet d'une dévotion particulièrement intense ont été et sont encore, dans bien des régions de l'Europe catholique, habillées de vêtements réels. La coutume réserve presqu'exclusivement à des femmes le droit de les changer, et donc de voir et de toucher le corps de la statue. L'Église, pourtant, n'accorde qu'aux prêtres le droit de manipuler les objets sacrés, et les femmes, on le sait, ne peuvent accéder à la prêtrise. Comment comprendre, par conséquent, que les «chambrières » de la Vierge soient des femmes ? Comment assument-elles leur fonction et quel sens lui donnent-elles?

The statues of the Virgin which are objects of a particularly intense devotion have been and still are, in many areas of Europe, dressing with real garments. According to tradition, only women are allowed to change them and, consequently, to see and touch the body of the statue. However, the Church grants to priests only the right to handle sacred objects ; and women, of course, are excluded from priesthood. Therefore, how can one explain the Virgin's «ladies in-waiting » are women? How do they fulfil this duty and what is its meaning for them? 


\section{AUTEUR}

\section{MARLĖNE ALBERT-LLORCA}

Marlène ALBERT-LLORCA est chercheur au Centre d'Anthropologie (CNRS-EHESS) de Toulouse. Après avoir publié L'ordre des choses. Les récits d'origine des animaux et des plantes en Europe. Paris, «Le regard de l'ethnologue », CTHS, 1991, un ouvrage consacré à l'étude des classifications populaires des êtres naturels, elle a centré ses recherches sur l'anthropologie du christianisme dans l'Europe du sud. Elles portent actuellement, plus précisément, sur le statut cognitif et social des images dites « miraculeuses » de la Vierge et sur les fêtes de « Maures et Chrétiens » de la région d'Alicante. Adresse : Centre d'Anthropologie. EHESS/CNRS. 56, rue du Taur, 31000 Toulouse. 